\title{
Bio-gasoline and Bio-kerosene Production by Fractional Distillation of Pyrolysis Bio-Oil Açaí Seeds
}

\author{
D. A. R. de Castro ${ }^{b}$; H. J. da Silva Ribeiro ${ }^{b}$; L. H. H. Guerreiro ${ }^{a}$; L. P. Bernar ${ }^{b}$; S. J. Bremer ${ }^{d}$; \\ H. da Silva Almeida ${ }^{c}$; M. C. Santos ${ }^{\text {a }}$; ;. Duvoisin Jr. ${ }^{\text {e }}$ L. E. P. Borges ${ }^{f}$ *N. T. Machado ${ }^{\text {b,c }}$ \\ ${ }^{a}$ Program of Chemical Engineering-UFPA \\ ${ }^{b}$ Graduate Program of Natural Resources Engineering-UFPA \\ ${ }^{c}$ Faculty of Sanitary and Environmental Engineering-UFPA \\ Rua Augusto Corrêia $N^{o}$ 1, CEP: 66075-900, Belém-Pará-Brazil \\ ${ }^{d}$ Hochschule für Technik und Wirtschaft Berlin \\ Wilhelminenhofstrasse 75A, 12459 Berlin-Germany \\ ${ }^{e}$ Faculty of Chemical Engineering-UEA \\ Avenida Darcy Vargas N. 1200, CEP: 69050-020 Manaus, Amazonas, Brazil \\ ${ }^{f}$ Laboratory of Catalyst Preparation and Catalytic Cracking \\ Section of Chemical Engineering-IME \\ Praça General Tibúrcio No. 80, CEP: 22290-270, Rio de Janeiro-RJ-Brazil
}

\section{Abstract}

The bio-oil obtained by pyrolysis of Açaí (Euterpe oleracea Mart.) seeds at $450^{\circ} \mathrm{C}, 1.0$ atmosphere, in technical scale, submitted to fractional distillation to produce biofuels-like fractions. The distillation of bio-oil carried out in a laboratory distillation column (Vigreux) of $30 \mathrm{~cm}$. The physical-chemistry properties (density, kinematic viscosity, acid value and refractive index) determined by official methods. The chemical functions present in distillation fractions determined by FT-IR and the chemical composition by GC-MS. The distillation of bio-oil yielded gasoline, light kerosene, and kerosene-like fuel fractions of 16.16, 19.56, and $41.89 \%$ (wt.), respectively. All the physical-chemistry properties (density, kinematic viscosity, acid value and refractive index) increase with boiling temperature. The gasoline-like fraction is composed by $64.0 \%$ (area.) hydrocarbons and $36.0 \%$ (area.) oxygenates, while light kerosenelike fraction by $66.67 \%$ (area.) hydrocarbons and 33.33\% (area.) oxygenates, and kerosenelike fraction by $19.87 \%$ (area.) hydrocarbons and $81.13 \%$ (area.) oxygenates.

Keywords: Açaí, Residual Seeds, Pyrolysis, Bio-Oil, Distillation, Bio-gasoline, Bio-kerosene.

\section{Introduction}

Açaí (Euterpe oleracea Mart.) is a native palm of natural occurrence in tropical Central and South America [1]. The palm gives a dark-purple, berry-like fruit, clustered into bunches [2]. The fresh fruits are traditionally processed by crushing and/or extracting the pulp and skin 
with warm water to produce a thick, purple-colored beverage/juice or a paste [3-4]. The fruit is a staple food in rural and urban areas of the Amazon River estuary, particularly in the State Pará (Pará-Brazil), with a great economic importance at both rural livelihoods and regional levels [5]. It has become one of the most important export products of the Amazon River estuary to other parts of Brazil [5], as well as oversees [6].

Of the total 1.228.811 tons/year of fruits produced by the State Pará, between 85\% [7] and $83 \%$ (wt.) [8], is a residue (Açaí seeds), thus producing between 1.019.913 and 1.044.489 tons/year of a residue. The mechanical processing of Açaí fruits in nature produces around 175.7 tons residue/day in off-season crop and 448.0 tons residue/day in the season crop in the metropolitan region of Belém (Pará-Brazil), posing a complex environmental problem of solid waste management $[9,10]$. The Açaí fruit is a small dark-purple, berry-like fruit, almost spherical, weighing between 2.6 to $3.0 \mathrm{~g}$ [11], with a diameter around 10.0 and $20.0 \mathrm{~mm}$ [11], containing a large core seed that occupies almost 85\% (vol./vol.) of its volume [3]. Açaí (Euterpe oleracea Mart.) fruit has an oily-fiber seed, rich in lignin-cellulose material [12-15].

Pyrolysis makes it possible the use of low quality lignin-cellulosic based material to produce not only liquid bio-oils, but also gaseous fuels, and a carbonaceous rich solid phase, as reported in the literature [16-73], and studies include biomass pyrolysis [23-24, 26, 45, 56-57, 62, 67-68], bio-oil chemical upgrading techniques [26, 45, 50], bio-oils physical-chemical properties $[21,25-26,28,34-35,43,57,62-63]$, as well as separation and/or purification processes to improve bio-oils quality [17-22, 30-33, 36-41, 46-48, 51, 53-54, 59-61, 65-66, 7073].

The bio-oil produced by pyrolysis is a multicomponent liquid mixture presenting water, carboxylic acids, aldehydes, ketones, alcohols, esters, ethers, aliphatic hydrocarbons, aromatic hydrocarbons, anhydrous-sugars, furans, phenols derivatives, among others chemical functions [16-17, 20, 38, 44, 47-48, 53, 60-61, 73]. In addition, its organic fraction has a wide distribution of polarity, molecular weight [47], as well as differences in thermo-physical and transport properties of chemical compounds, as reported by the simulation of organic liquid compounds [74], posing challenges to the efficient separation and/or purification processes [47, 74].

In the last years, several thermal and physical separation processes were applied to remove oxygenates from biomass-derived bio-oils including molecular distillation [30, 33, 36$39,71]$, fractional distillation $[17-21,40-41,46-48,53,59-60,66,70,72-73]$, liquid-liquid extraction [22, 31, 61], and fractional condensation [51, 54, 65]. In addition, chemical methods such as catalytic upgrading of bio-oils vapors have been applied to improve bio-oils quality [19, $29,64]$. 
The fractional distillation studies were carried out in micro/bench scale [17, 46-47], laboratory scale $[41,53,66,70,72-73]$, and pilot scale [21], under atmospheric [17-18, 46-48, 53, 66, 70, 72-73], or under vacuum [18-19, 41, 48, 53]. Açaí (Euterpe oleracea, Mart.) seeds are the only fruit specie, whose centesimal and elemental composition is completely different from wood biomass (aspen poplar wood, eucalyptus, maple wood, and softwood bark) [17-19, 21, 53], agriculture residues of cereal grains (corn Stover, rice Rusk) [41, 46-47, 66, 70, 72], jatropha curcas [46], and until horse manure and switch-grass [53]. However, until the moment no systematic study investigated the physicochemical properties (density, kinematic viscosity, refractive index, and acid value) chemical composition of Açaí (Euterpe oleracea, Mart.) seeds bio-oil distillation fractions [73].

In this work, fractional distillation of bio-oil obtained by pyrolysis of Açaí seeds at 450 ${ }^{\circ} \mathrm{C}, 1.0$ atmosphere, in technical scale, has been investigated systematically using a laboratoryscale column (Vigreux) to produce fuels-like fractions (gasoline, light kerosene, and kerosene), as well as to determine the physical-chemistry properties (density, kinematic viscosity, acid value and refractive index) and chemical composition of distillation fractions.

\section{Materials and methods}

\subsection{Materials, pre-treatment, and characterization of Açai (Euterpe oleracea, Mart.)} seeds in nature

The seeds of Açaí (Euterpe oleracea Mart.) in nature obtained in a small store of Açaí commercialization, located in the City of Belém-Pará-Brazil [73]. The seeds were submitted to pre-treatments of drying and grinding as reported elsewhere [73]. The dried and grinded seeds were physical-chemistry characterized for moisture, volatile matter, ash, fixed carbon, lipids, proteins, fibers, and insoluble lignin according to official methods $[73,75,76]$.

\subsection{Fractional distillation of bio-oil}

\subsubsection{Distillation: Experimental apparatus and procedures}

The fractional distillation of bio-oil was performed by using an experimental apparatus and procedures described elsewhere [73, 77-78]. The aqueous phase presented in the distillation fractions was separated from the organic phase by decantation using a $250 \mathrm{ml}$ glass separator funnel. Afterwards, filtration was applied to remove small solid particles present in the organic phase.

\subsection{Physical-chemistry analysis and chemical composition of distillation fractions}

\subsubsection{Physical-chemistry analysis of distillation fractions}

The distillation fractions were (gasoline, light kerosene, and kerosene) physicalchemistry characterized for acid value (AOCS Cd 3d-63), density (ASTM D4052) at $25^{\circ} \mathrm{C}$, 
kinematic viscosity (ASTM D445/D446) at $40^{\circ} \mathrm{C}$, and refractive index (AOCS Cc 7-25) [81]. The analysis of chemical functions (carboxylic acids, aliphatic and aromatic hydrocarbons, ketones, phenols, aldehydes, furans, esters, ethers, etc.) present in distillation fractions determined by FT-IR [73, 77].

\subsubsection{Chemical composition of distillation fractions}

The chemical composition of distillation fractions determined by CG-MS and the equipment and operational procedures described in details elsewhere [73].

\section{Results and discussions}

\subsection{Material balances and yields of fractional distillation}

Table 1 summarizes the material balance and yields by fractional distillation of bio-oil, and the distillation fractions and bottoms are illustrated in Figure 1. The yields of fuel-like fractions (gasoline, light kerosene, and kerosene) were 16.16, 19.56, and 41.89\% (wt.), respectively, giving a total distillation yield of $77.61 \%$ (wt.). The results are according to similar studies for distillation of biomass derived bio-oil in the literature [17-19, 21, 41, 46-48, 53, 66, $70,72]$. The yield of distillation fractions, is higher than those reported in the literature for both atmospheric and vacuum conditions [17-19, 21, 41, 46-48, 53, 66, 70, 72].

Table 1: Material balance and yields by fractional distillation of bio-oil.

\begin{tabular}{|c|c|c|c|c|c|c|c|c|c|c|c|c|c|}
\hline Distillation: & Bio-Oil & Gas & Raffinate & \multicolumn{5}{|c|}{ Distillates [g] } & \multicolumn{5}{|c|}{ Yield [wt.\%] } \\
\hline Vigreux Column & [g] & {$[g]$} & [g] & $\mathrm{H}_{2} \mathrm{O}$ & G & LK & K & LD & $\mathrm{H}_{2} \mathrm{O}$ & G & LK & $\mathbf{K}$ & LD \\
\hline$\left(30^{\circ} \mathrm{C}-215^{\circ} \mathrm{C}\right)$ & 307.53 & 0 & 69.87 & 0 & 49.48 & 59.91 & 128.27 & 0 & 0 & 16.16 & 19.56 & 41.89 & 0 \\
\hline
\end{tabular}

G = Gasoline, $\mathbf{L K}=$ Light Kerosene, $\mathrm{K}=$ Kerosene, $\mathbf{L D}=$ Light Diesel.

Zheng and Wei [41] reported by distillation of fast pyrolysis bio-oil at $80^{\circ} \mathrm{C}$ under vacuum (15 mmHg), a distilled bio-oil yield of 61\% (wt.). Zhang et. al. [47] reported by atmospheric distillation of fast pyrolysis bio-oil, an accumulated distillate of $51.86 \%$ (wt.). Zhang et. al. [47] observed that as the distillation temperature reached $240^{\circ} \mathrm{C}$, condensation reactions take place, generating water, a behavior not observed during the course of distillation as illustrated in Table 1. Capunitan and Capareda [48] reported for the distillation at atmospheric condition, an organic phase (Distillates) yield of $15.0 \%$ (wt.) at $100^{\circ} \mathrm{C}, 4.7 \%$ (wt.) between $100^{\circ} \mathrm{C}<\mathrm{T}^{\text {Boiling }}<180^{\circ} \mathrm{C}$, and $45.3 \%$ (wt.) between $180^{\circ} \mathrm{C}<\mathrm{T}^{\text {Boiling }}<250^{\circ} \mathrm{C}$, while vacuum distillation yielded $10.3 \%$ (wt.) of an organic phase at $80^{\circ} \mathrm{C}, 5.9 \%$ (wt.) between $80^{\circ} \mathrm{C}$ $<\mathrm{T}^{\text {Boiling }}<160^{\circ} \mathrm{C}$, and $40.9 \%$ (wt.) between $160^{\circ} \mathrm{C}<\mathrm{T}^{\text {Boiling }}<230^{\circ} \mathrm{C}$. Elkasabi et. al. [53] reported by distillation of tail-gas reactive pyrolysis (TGRP) bio-oil, yields ranging from 55 to $65 \%$ (wt.). 


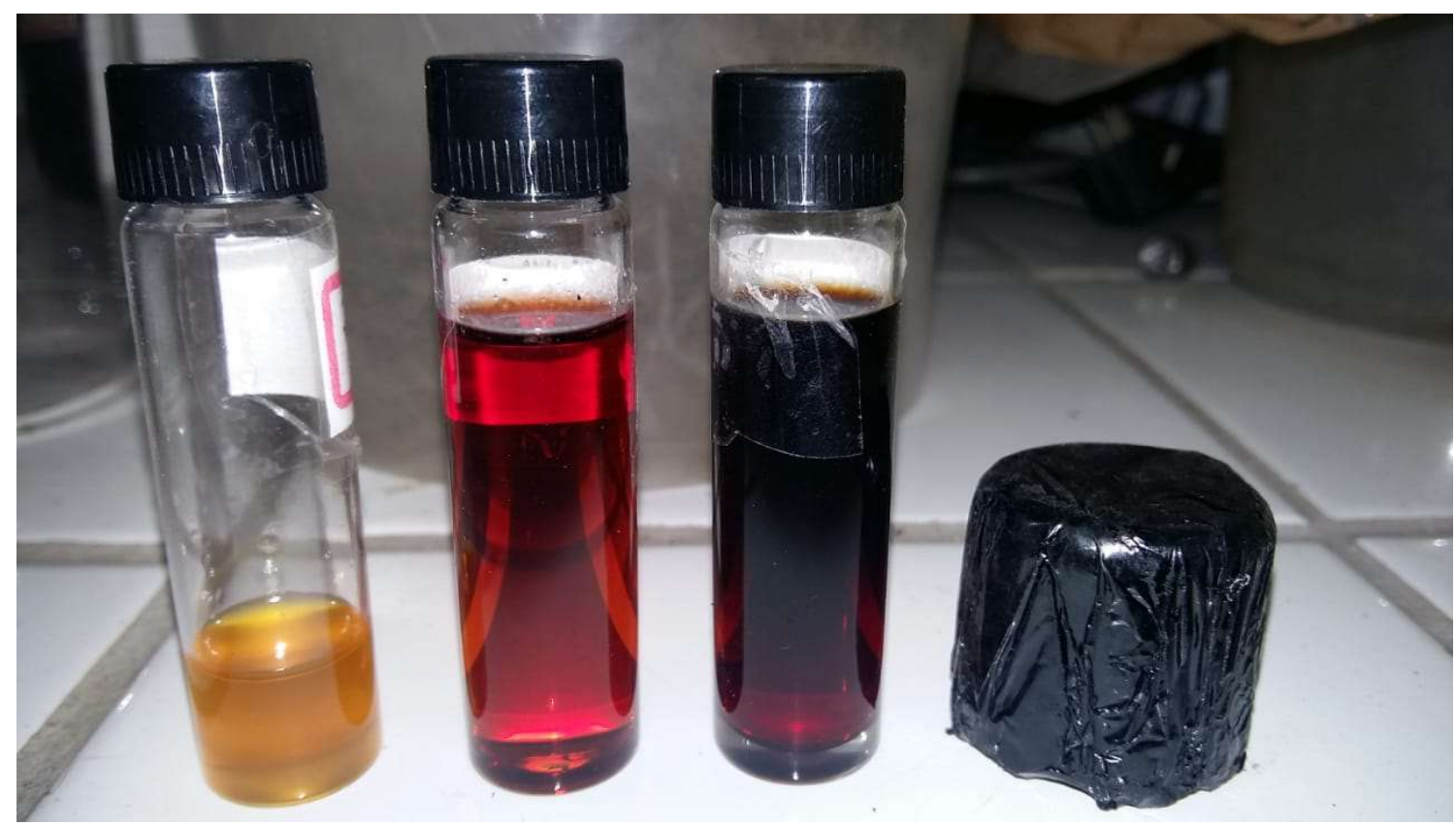

Figure 1: Distillation fractions [gasoline (yellow), light kerosene (red), and kerosene (red dark)like boiling range temperature fossil fuels] and bottoms [Raffinate (black solid)] obtained by fractional distillation of bio-oil produced by pyrolysis of Açaí (Euterpe oleracea, Mart.) seeds at $450{ }^{\circ} \mathrm{C}$ and 1.0 atmosphere, in pilot scale.

\subsection{Physical-chemical properties of distillation fractions}

The physical-chemical properties of distillation fractions (gasoline, $80-175^{\circ} \mathrm{C}$; light kerosene, $175-200^{\circ} \mathrm{C}$; and kerosene-like fraction, $200-215^{\circ} \mathrm{C}$ ) of bio-oil are illustrated in Table 2 .

Table 2: Physical-chemical properties of distillation fractions of bio-oil.

\begin{tabular}{l|lll|l}
\hline \multirow{2}{*}{$\begin{array}{c}\text { Physico- } \\
\text { chemical } \\
\text { Properties }\end{array}$} & \multicolumn{3}{|c|}{$\mathbf{4 5 0}^{\circ} \mathbf{C}$} & \multirow{2}{*}{ ANP N } \\
\cline { 2 - 4 } & G & LK & K & \multirow{2}{*}{$\mathbf{0 . 8 2 - 0 . 8 5}$} \\
\hline$\rho\left[\mathrm{g} / \mathrm{cm}^{3}\right], 30^{\circ} \mathrm{C}$ & 0.9146 & 0.9191 & 0.9816 & \\
I. A $[\mathrm{mg} \mathrm{KOH} / \mathrm{g}]$ & 14.94 & 61.08 & 64.78 & \\
I. R[-] & 1.455 & 1.479 & 1.497 & \\
$v\left[\mathrm{~mm}^{2} / \mathrm{s}\right], 40^{\circ} \mathrm{C}$ & 1.457 & 3.106 & 4.040 & $\mathbf{2 . 0 - 4 . 5}$ \\
\hline
\end{tabular}

I.A=Acid Value, I.R=Refractive Index.

It can be observed that acidity of distillation fractions increases with boiling temperature. However, the acidity of gasoline-like fraction is much lower than that of raw biooil $(70.26 \mathrm{mg} \mathrm{KOH} / \mathrm{g})$, as described in Table 3. The high acid value of bio-oil is due to the 
presence of $78.48 \%$ (area.) oxygenates, as shown in Table 4 . The same behavior was observed for the densities, kinematic viscosities, and refractive indexes of gasoline, light kerosene, and kerosene-like like fractions with increasing boiling temperature. This is probably due to the high concentration of higher-boiling-point compounds in the distillate fractions, such as phenols, cresols ( $p$-cresol, $o$-cresol), and furans, as the concentration of those compounds in the distillation fractions increases with increasing boiling temperature as reported elsewhere [66, 70, 72], corroborate in Tables 5, 6, and 7.

The gasoline, light-kerosene, and kerosene-like fuel densities were 0.9146, 09191, and $0.9816 \mathrm{~g} / \mathrm{mL}$. The gasoline-like fuel density (fractions $\left(40^{\circ} \mathrm{C}<\mathrm{T}^{\text {Boiling }}<175^{\circ} \mathrm{C}\right)$, higher, but close to the density of distillation fraction of $0.8733 \mathrm{~g} / \mathrm{mL}\left(\mathrm{T}^{\text {Boiling }}<140^{\circ} \mathrm{C}\right)$ for jatropha curcas cake pyrolysis bio-oil reported by Majhi et. al. [46]. This is probably due to the high lipids content between $14-18 \%$ (wt.) and 10-10.9\% (wt.) fiber, thus producing a bio-oil similar to lipid-based pyrolysis organic liquid products [77-78]. The gasoline, light-kerosene, and kerosene-like fuel kinematic viscosities were $1.457,3.106$, and $4.040 \mathrm{~mm}^{2} / \mathrm{s}$, lower than the distillation fraction kinematic viscosity of $2.350 \mathrm{~mm}^{2} / \mathrm{s}\left(\mathrm{T}^{\text {Boiling }}<140^{\circ} \mathrm{C}\right)$ for jatropha curcas cake pyrolysis bio-oil reported by Majhi et. al. [46].

The acid value of gasoline, light-kerosene, and kerosene-like fuel fractions were 14.94, 61.08 , and $64.78 \mathrm{mg} \mathrm{KOH} / \mathrm{g}$, lower than the distillation fraction acid value of $0.05 \mathrm{mg} \mathrm{KOH} / \mathrm{g}$ ( $\mathrm{T}^{\text {Boiling }}<140^{\circ} \mathrm{C}$ ) for jatropha curcas cake pyrolysis bio-oil distillation reported by Majhi et. al. [46], the organic phases (distillates) acid values of $4.1\left(100^{\circ} \mathrm{C}<\mathrm{T}^{\text {Boiling }}\right), 15.1\left(100^{\circ} \mathrm{C}<\mathrm{T}^{\text {Boiling }}\right.$ $\left.<180^{\circ} \mathrm{C}\right)$, and $7.41\left(180^{\circ} \mathrm{C}<\mathrm{T}^{\text {Boiling }}<250^{\circ} \mathrm{C}\right) \mathrm{mg} \mathrm{KOH} / \mathrm{g}$, for corn Stover bio-oil atmospheric distillation reported by Capunitan and Capareda [48], the organic phases (distillates) acid values of $3.0\left(80^{\circ} \mathrm{C}<\mathrm{T}^{\text {Boiling }}\right), 13.9\left(80^{\circ} \mathrm{C}<\mathrm{T}^{\text {Boiling }}<160^{\circ} \mathrm{C}\right)$, and $5.0\left(160^{\circ} \mathrm{C}<\mathrm{T}^{\text {Boiling }}<230^{\circ} \mathrm{C}\right) \mathrm{mg}$ $\mathrm{KOH} / \mathrm{g}$, for corn Stover bio-oil vacuum distillation reported by Capunitan and Capareda [48], the acid values of $13.5 \mathrm{mg} \mathrm{KOH} / \mathrm{g}\left(\mathrm{T}^{\text {Boiling }}=192^{\circ} \mathrm{C}\right)$ and $5.3 \mathrm{mg} \mathrm{KOH} / \mathrm{g}\left(\mathrm{T}^{\text {Boiling }}=220^{\circ} \mathrm{C}\right)$ of distillation fractions $\mathrm{F}_{3}$ and $\mathrm{F}_{4}$ of $\mathrm{TGRP}_{1}$, and the acid value of $11.1 \mathrm{mg} \mathrm{KOH} / \mathrm{g}\left(\mathrm{T}^{\text {Boiling }}=\right.$ $235^{\circ} \mathrm{C}$ ) of distillation fraction $\mathrm{F}_{5}$ of $\mathrm{TGRP}_{2}$, for tail-gas reactive pyrolysis of horse manure (TGRP 1$)$, switch grass $\left(\mathrm{TGRP}_{2}\right)$, and eucalyptus $\left(\mathrm{TGRP}_{3}\right)$, reported by Elkasabi et. al. [53].

The results reported by Elkasabi et. al. [53], show that fractional distillation was not effective to diminish the acid values of TGRP bio-oil with initial high acid values, what does not agree with the results reported by Capunitan and Capareda [48], as well as those presented in Table 2, showing that the acid values of distillation fractions are lower than that of raw biooil, proving that distillation was effective. 
Table 3: Physical-chemical properties of bio-oil, compared to similar studies reported in the literature $[21,25,28,47,69,79-80]$.

\begin{tabular}{l|l|l|l|l|l|l|l|l|l}
\hline \multirow{2}{*}{$\begin{array}{c}\text { Physicochemical } \\
\text { Properties }\end{array}$} & $\mathbf{4 5 0}{ }^{\circ} \mathrm{C}$ & {$[\mathbf{2 1}]$} & {$[\mathbf{2 5}]$} & {$[\mathbf{2 8}]$} & {$[\mathbf{4 7}]$} & {$[\mathbf{6 9}]$} & {$[\mathbf{7 9}]$} & {$[\mathbf{8 0}]$} & \\
\cline { 2 - 7 } & Bio-Oil & Bio-Oil & Bio-Oil & Bio-Oil & Bio-Oil & Bio-Oil & Bio-Oil & Bio-Oil & \\
\hline$\rho\left[\mathrm{g} / \mathrm{cm}^{3}\right], 30^{\circ} \mathrm{C}$ & $\mathbf{1 . 0 4 3}$ & $\mathbf{1 . 0 6 6}$ & 1.250 & 1.140 & 1.190 & 1.1581 & 1.200 & $\mathbf{1 . 0 3 0}$ & $\mathbf{0 . 8 2 - 0 . 8 5}$ \\
I. A $[\mathrm{mg} \mathrm{KOH} / \mathrm{g}]$ & 70.26 & - & - & - & - & - & - & & - \\
I. R $[-]$ & $\mathrm{ND}$ & - & - & - & - & - & - & & - \\
$v\left[\mathrm{~mm}^{2} / \mathrm{s}\right], 40^{\circ} \mathrm{C},{ }^{*} 60^{\circ} \mathrm{C}$ & 68.34 & 38.0 & 148.0 & 13.2 & $40.0^{*}$ & $5.0-13.0$ & 12.0 & - & $\mathbf{2 . 0}$ \\
\hline
\end{tabular}

I.A = Acid Value; I.R = Refractive Index; ANP: Brazilian National Petroleum Agency, Resolution $\mathbf{N}^{\circ} 65$ (Specification of Diesel S10); ND = Not Determined.

\subsection{FT-IR and GC-MS analyses of bio-oil and distillation fractions}

\subsubsection{FT-IR spectroscopy of bio-oil and distillation fractions}

By the FT-IR analysis of bio-oil and distillation fractions (gasoline: $40-175{ }^{\circ} \mathrm{C}$, light kerosene: $175-200{ }^{\circ} \mathrm{C}$, and kerosene-like fraction: $200-215^{\circ} \mathrm{C}$ ), summarized in Figure 2, the identification of absorption bands/peaks was performed according to previous studies $[28,48$, $59,73,77-78,80,81]$. The FT-IR spectroscopy of bio-oil and distillation fraction identify the presence of hydrocarbons (alkanes, alkenes, and aromatic hydrocarbons) and oxygenates (phenols, cresols, carboxylic acids, alcohols, ethers, ketones, and furans).

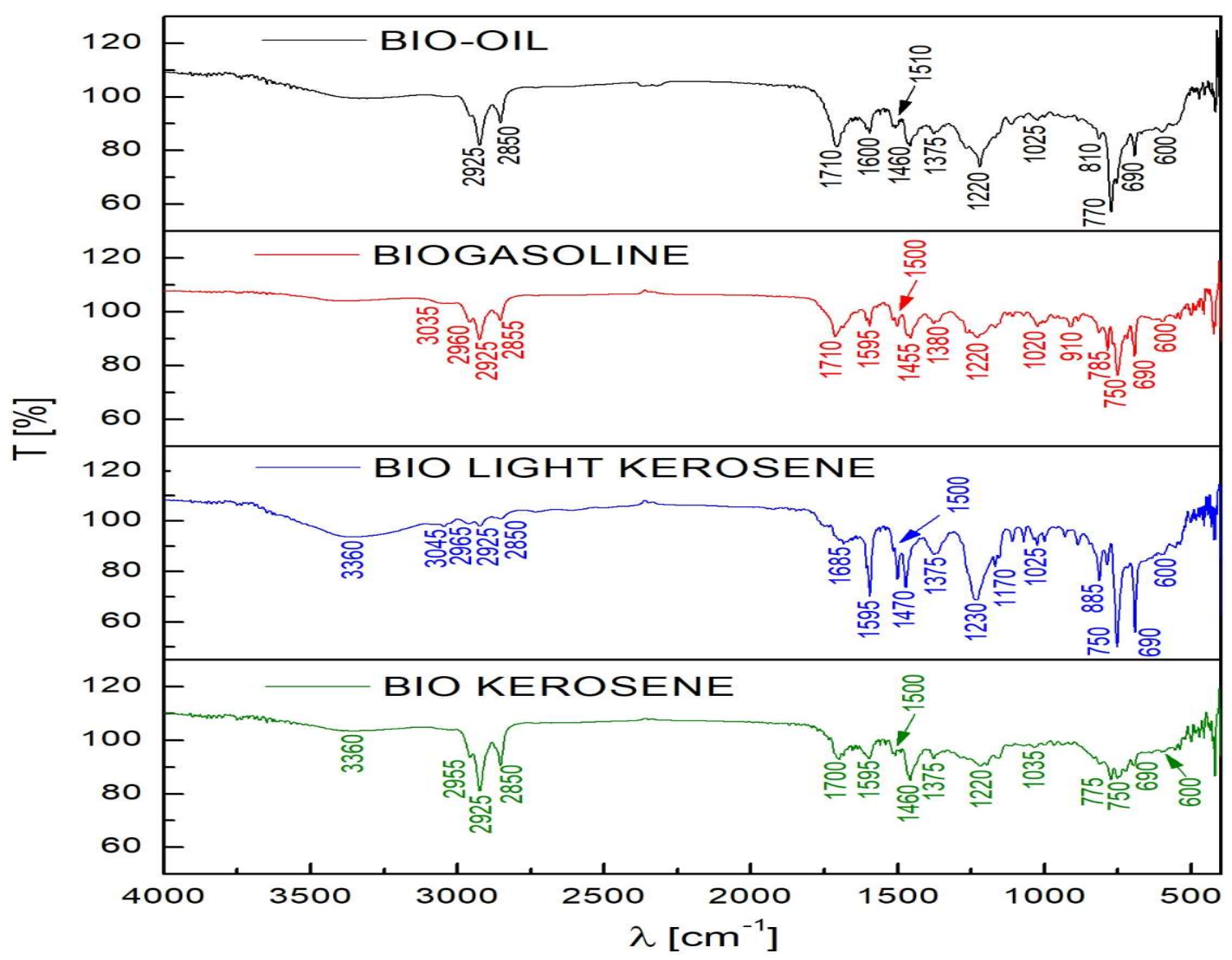

Figure 2: FT-IR of Açaí seeds bio-oil and distillation fractions. 


\subsubsection{Chemical compositional of bio-oil and distillation fractions by GC-MS}

\subsubsection{Chemical compositional of bio-oil by GC-MS}

The chromatogram of bio-oil is shown in Figure 3. The peaks are concentrated between retention times of 8.0 and 22.0 minutes, with the highest one around 12.5 minutes. The GC-MS identified hydrocarbons (alkanes, alkenes, aromatic hydrocarbons, and cycloalkenes) and oxygenates (esters, phenols, cresols, carboxylic acids, ketones, furans, and aldehydes) in biooil, being composed of $21.52 \%$ (area.) hydrocarbons and $78.48 \%$ (area.) oxygenates [73]. The high acidity of bio-oil, described in Table 3, is probably due to the presence of carboxylic acids, ketones, aldehydes, phenols and cresols confer the high acidity of bio-oil.

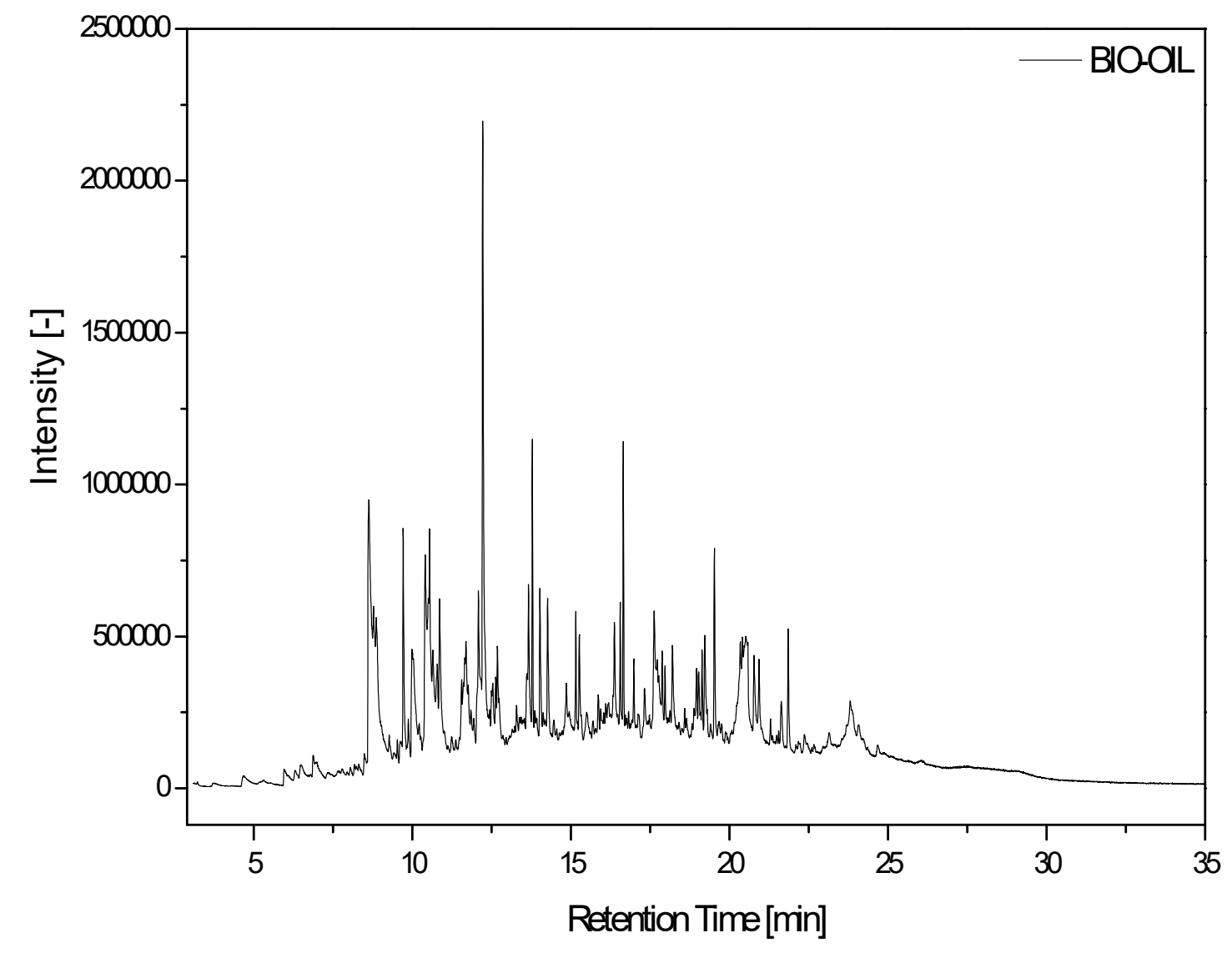

Figure 3: GC-MS of bio-oil.

The composition of bio-oil shows similarity to those reported in the literature [27, 34, 41, 47-48, 53, 61], showing the presence of hydrocarbons, phenols, cresols, furans, carboxylic acids, and esters, among other classes of compounds [73]. The identification of hydrocarbons with carbon chain length between $\mathrm{C}_{11}$ and $\mathrm{C}_{15}$, shows the presence of heavy gasoline 
compounds with $\mathrm{C}_{11}\left(\mathrm{C}_{5}-\mathrm{C}_{11}\right)$, light kerosene-like fractions $\left(\mathrm{C}_{11}-\mathrm{C}_{12}\right)$, and light diesel-like fractions $\left(\mathrm{C}_{13}-\mathrm{C}_{15}\right)$, according to Table 4 .

Table 4: Classes of compounds, summation of peak areas, CAS number, and retention times of chemical compounds identified by CG-MS in bio-oil.

\begin{tabular}{|c|c|c|c|}
\hline Class of Compounds: Chemical Compounds & $\mathrm{RT}[\mathrm{min}]$ & CAS & $\omega_{\mathrm{i}} \%$ (Area) \\
\hline \multicolumn{4}{|l|}{ Alkanes } \\
\hline Undecane & 10.622 & $1120-21-4$ & 1.124 \\
\hline Tridecane & 13.870 & $629-50-5$ & 2.481 \\
\hline Pentadecane & 16.744 & $629-62-9$ & 2.290 \\
\hline Dodecane, 5,8-diethyl & 19.326 & $24251-86-3$ & 1.626 \\
\hline$\Sigma($ Area. $\%)=$ & & & 7.521 \\
\hline \multicolumn{4}{|l|}{ Alkenes } \\
\hline 6-Tridecene, (Z)- & 1.626 & $6508-77-6$ & 2.118 \\
\hline$\Sigma($ Area. $\%)=$ & & & 2.118 \\
\hline \multicolumn{4}{|l|}{ Cycloalkenes } \\
\hline Megastigma-4,6(E), $8(Z)$-trien & 13.440 & $5298-13-5$ & 1.847 \\
\hline$\Sigma($ Area. $\%)=$ & & & 1.847 \\
\hline \multicolumn{4}{|l|}{ Aromatic Hydrocarbons } \\
\hline Naphthalene & 12.262 & $91-20-3$ & 4.399 \\
\hline Naphthalene, 1-methyl & 14.046 & $90-12-0$ & 2.390 \\
\hline 1H-Indene, 1-ethylidene & 14.296 & $2471-83-2$ & 3.249 \\
\hline$\Sigma($ Area. $\%)=$ & & & 10.038 \\
\hline \multicolumn{4}{|l|}{ Esters } \\
\hline Undecanoic acid, 10-methyl-, methyl ester & 17.049 & $5129-56-6$ & 1.096 \\
\hline Methyl tetradecanoate & 19.620 & $124-10-7$ & 2.969 \\
\hline$\Sigma($ Area. $\%)=$ & & & 4.065 \\
\hline \multicolumn{4}{|l|}{ Carboxylic Acids } \\
\hline Dodecanoic acid & 17.648 & $334-48-5$ & 4.307 \\
\hline Tetradecanoic acid & 20.677 & $544-63-8$ & 4.216 \\
\hline$\Sigma($ Area.\% $)=$ & & & 8.523 \\
\hline \multicolumn{4}{|l|}{ Ketones } \\
\hline 2-Pentanone, 4-hydroxy-4-methyl & 5.886 & $123-42-2$ & 1.878 \\
\hline 2-Cyclopenten-1-one, 2,3-dimethyl & 9.552 & $1121-05-7$ & 1.655 \\
\hline$\Sigma($ Area.\% $\%)=$ & & & 3.533 \\
\hline \multicolumn{4}{|l|}{ Phenols } \\
\hline Phenol & 8.469 & $108-95-2$ & 15.932 \\
\hline Phenol, 2-methoxy & 10.446 & 90-05-1 & 4.583 \\
\hline Phenol, 2,6-dimethyl & 10.805 & $576-26-1$ & 1.991 \\
\hline Phenol, 2,4-dimethyl & 11.469 & $105-67-9$ & 2.034 \\
\hline Phenol, 2,5-dimethyl & 11.502 & $95-87-4$ & 2.215 \\
\hline Phenol, 3,4-dimethyl & 11.821 & $95-65-8$ & 3.845 \\
\hline Phenol, 4-ethyl-2-methoxy & 13.571 & 2785-89-9 & 4.567 \\
\hline$\Sigma($ Area. $\%)=$ & & & 35.167 \\
\hline \multicolumn{4}{|l|}{ Cresols } \\
\hline p-Cresol & 9.818 & $108-39-4$ & 6.331 \\
\hline m-Cresol & 10.198 & $106-44-5$ & 11.054 \\
\hline Cresol & 12.210 & $93-51-3$ & 3.141 \\
\hline$\Sigma($ Area. $\%)=$ & & & 20.526 \\
\hline \multicolumn{4}{|l|}{ Furans } \\
\hline Benzofuran, 2-methyl & 10.879 & $4265-26-2$ & 1.879 \\
\hline Furan, 2-(2 furanylmethyl)-5-methyl & 11.946 & $13678-51-8$ & 2.089 \\
\hline Benzofuran, 4,7-dimethyl & 12.700 & $28715-26-6$ & 1.783 \\
\hline$\Sigma($ Area. $\%)=$ & & & 5.751 \\
\hline \multicolumn{4}{|l|}{ Aldehyds } \\
\hline Cinnamaldehyde, $\beta$-methyl- & 12.654 & $1196-67-4$ & 0.910 \\
\hline$\Sigma($ Area. $\%)=$ & & & 0.910 \\
\hline
\end{tabular}




\subsubsection{Chemical compositional of distillation fractions by GC-MS}

The chromatogram of bio-oil distillation fractions (gasoline: $40-175^{\circ} \mathrm{C}$, light kerosene: $175-200^{\circ} \mathrm{C}$, and kerosene-like fraction: $200-215^{\circ} \mathrm{C}$ ) are shown in Figures 4, 5, and 6, respectively. One observes that the spectrum of peaks is moving to the right, showing that distillation was effective to fractionate the bio-oil.

The GC-MS identified in gasoline-like fraction hydrocarbons (alkanes, alkenes, and aromatic hydrocarbons) and oxygenates (esters, phenols, alcohols, ketones, furans, and aldehydes). The gasoline-like fraction contains $64.0 \%$ (area.) hydrocarbons (13.27\% alkenes, $9.41 \%$ alkanes, and $41.32 \%$ aromatic hydrocarbons) and $36.0 \%$ (area.) oxygenates $(5.50 \%$ esters, $2.61 \%$ ketones, $1.35 \%$ phenols, $6.05 \%$ alcohols, $13.24 \%$ furans, and $7.25 \%$ aldehydes). The absence of carboxylic acids confers the low acidity of gasoline-like fraction, as summarized in Table 5.

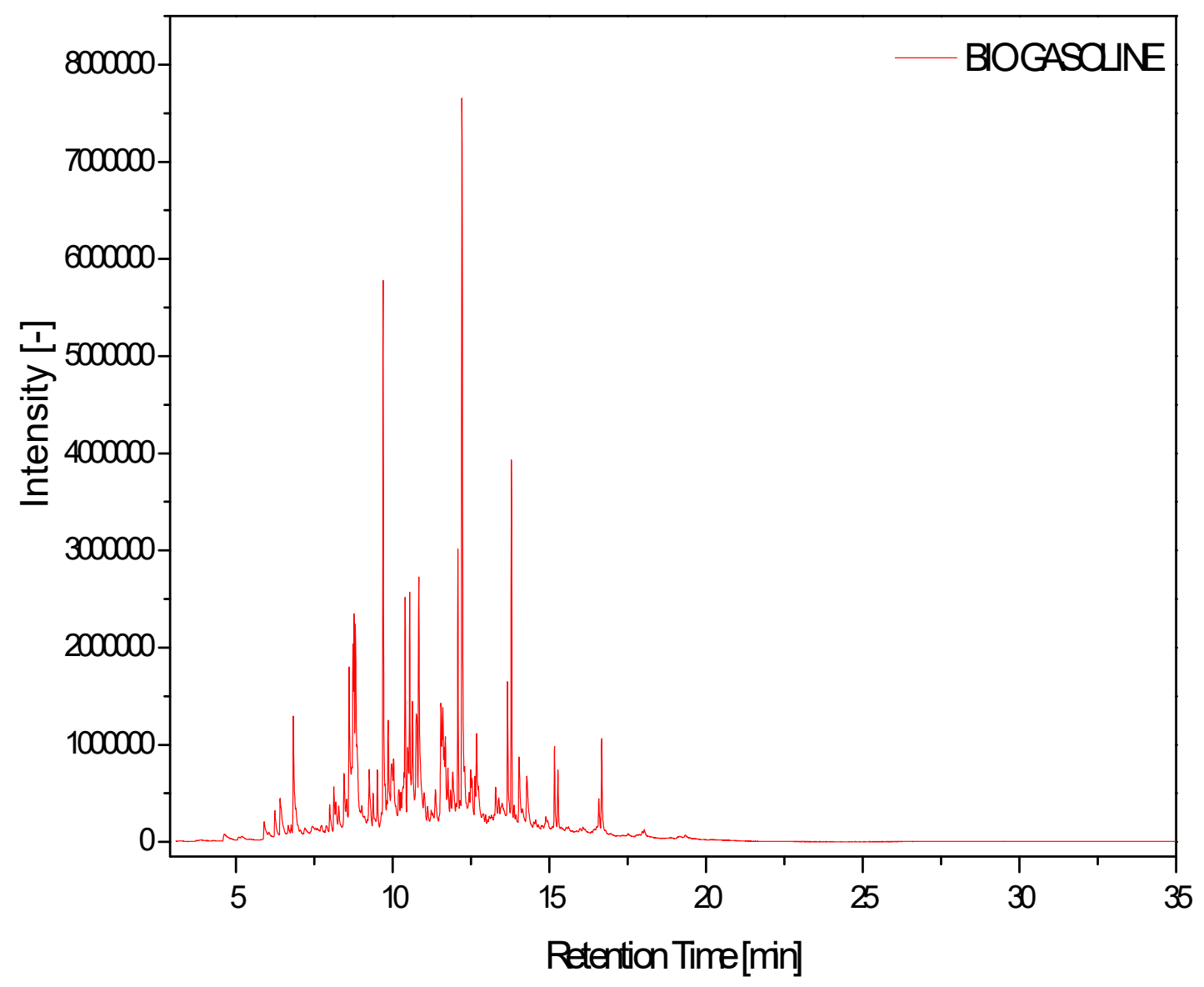

Figure 4: GC-MS of gasoline-like fraction $\left(40^{\circ} \mathrm{C}-175^{\circ} \mathrm{C}\right)$. 
Table 5: Classes of compounds, summation of peak areas, CAS number, and retention times of chemical compounds identified by CG-MS in gasoline-like fraction $\left(40^{\circ} \mathrm{C}-175^{\circ} \mathrm{C}\right)$.

\begin{tabular}{|c|c|c|c|}
\hline Class of Compounds: Chemical Compounds & $\mathrm{RT}$ [min] & CAS & $\omega_{\mathrm{i}} \%$ (Area) \\
\hline \multicolumn{4}{|l|}{ Alkanes } \\
\hline Undecane & 10.548 & $1120-21-4$ & 3.19 \\
\hline Tridecane & 13.794 & $629-50-5$ & 3.93 \\
\hline Tetradecane & 15.276 & $629-59-4$ & 0.75 \\
\hline Pentadecane & 16.744 & $629-62-9$ & 1.55 \\
\hline$\sum($ Area. $\%)=$ & & & 9.41 \\
\hline \multicolumn{4}{|l|}{ Alkenes } \\
\hline p-Mentha-1,5,8-triene & 9.861 & $21195-59-5$ & 2.254 \\
\hline 1-Undecene & 10.402 & $821-95-4$ & 2.776 \\
\hline 1-Dodecene & 12.088 & $112-41-4$ & 3.034 \\
\hline Bicyclo[6.4.0]dodeca-9,11-diene & 13.291 & - & 0.614 \\
\hline 1-Tridecene & 13.672 & $2437-56-1$ & 2.098 \\
\hline Bicyclo[4.4.1]undeca-1,3,5,7,9-pentaene & 14.286 & $2443-46-1$ & 1.380 \\
\hline 1-Tetradecene & 15.167 & $1120-36-1$ & 1.111 \\
\hline$\sum($ Area. $\%)=$ & & & 13.267 \\
\hline \multicolumn{4}{|l|}{ Aromatic Hydrocarbons } \\
\hline Benzene, 1,3-dimethyl- & 6.247 & $108-38-3$ & 0.578 \\
\hline Benzene, propyl- & 7.995 & $103-65-1$ & 0.516 \\
\hline Benzene, 1-ethyl-3-methyl- & 8.128 & $620-14-4$ & 0.686 \\
\hline Benzene, 1-ethyl-2-methyl- & 8.193 & $611-14-3$ & 0.593 \\
\hline Trimethylbenzene & 8.283 & $108-67-8$ & 0.566 \\
\hline Benzene, (1-methylethyl)- & 8.454 & $98-82-8$ & 1.050 \\
\hline Benzene, 1,2,4-trimethyl- & 8.738 & $95-63-6$ & 2.107 \\
\hline Benzene, 1-ethenyl-2-methyl- & 8.770 & $611-15-4$ & 2.709 \\
\hline Benzene, 1,2,3-trimethyl- & 9.255 & $526-73-8$ & 1.297 \\
\hline Benzene, pentyl- & 11.607 & $538-68-1$ & 2.205 \\
\hline Benzene, (1-methyl-2-propynyl)- & 11.646 & $4544-28-9$ & 0.875 \\
\hline Benzene, (1-methyl-2-cyclopropen-1-yl)- & 11.685 & 65051-83-4 & 1.441 \\
\hline o-Xylene & 6.413 & $95-47-6$ & 1.136 \\
\hline p-Xylene & 6.834 & $106-42-3$ & 2.080 \\
\hline 6,7-Dimethyl-3,5,8,8a-tetrahydro-1H-2-benzopyran & 10.368 & $110028-10-9$ & 1.243 \\
\hline 2,4-Dimethylstyrene & 11.371 & $2234-20-0$ & 0.703 \\
\hline 1H-Indene, 1-methyl- & 11.547 & $767-58-8$ & 1.830 \\
\hline Naphthalene & 12.217 & $91-20-3$ & 10.081 \\
\hline 1H-Indene, 2,3-dihydro-4,7-dimethyl- & 12.533 & $6682-71-9$ & 0.760 \\
\hline Benzocycloheptatriene & 14.038 & $264-09-5$ & 1.401 \\
\hline Indane & 9.516 & $496-11-7$ & 0.763 \\
\hline Indene & 9.699 & $95-13-6$ & 6.702 \\
\hline$\sum($ Area.\% $\%)=$ & & & 41.322 \\
\hline \multicolumn{4}{|l|}{ Esters } \\
\hline Hexanoic acid, 2-phenylethyl ester & 6.917 & $72934-12-4$ & 0.494 \\
\hline 2-Furancarboxylic acid, 3-phenylpropyl ester & 8.536 & - & 0.645 \\
\hline Carbonic acid, octadecyl phenyl ester & 8.616 & - & 3.193 \\
\hline $\begin{array}{l}\text { Acetic acid, 2-methylene-bicyclo[3.2.1] oct-6-en-8-yl } \\
\text { ester }\end{array}$ & 9.379 & - & 0.644 \\
\hline $\begin{array}{l}\text { 1-hydroxy-1,2,3,4-tetrahydronaphthalene } \\
\text { trifluoroacetate ester }\end{array}$ & 11.850 & $134563-46-5$ & 0.526 \\
\hline$\sum($ Area. $\%)=$ & & & 5.502 \\
\hline \multicolumn{4}{|l|}{ Ketones } \\
\hline 5H-Inden-5-one, 1,2,3,6,7,7a-hexahydro- & 9.975 & $1489-28-7$ & 1.630 \\
\hline $\begin{array}{l}\text { Tricyclo[4.2.1.1(2,5)]deca-3,7-dien-9-one, 10-hydroxy-10- } \\
\text { methyl- }\end{array}$ & 11.767 & $70220-88-1$ & 0.983 \\
\hline$\sum($ Area.$\%)=$ & & & 2.613 \\
\hline \multicolumn{4}{|l|}{ Phenols } \\
\hline Phenol & 8.704 & $108-95-2$ & 0.741 \\
\hline 2-(2-Hydroxyphenyl)buta-1,3-diene & 12.450 & $90-05-1$ & 0.608 \\
\hline$\sum($ Area. $\%)=$ & & & 1.349 \\
\hline Alcohols & & & \\
\hline
\end{tabular}


2-heptanol

1-Hexadecanol, 2-methyl-

Carveol

2-Indanol

2,6,8-Trimethylbicyclo[4.2.0]oct-2-ene-1,8-diol

1-Naphthalenol, 1,2,3,4-tetrahydro-3-methyl-

2-Naphthalenol, 1,2-dihydro-, acetate $\backslash 3$ -

Methoxymethoxy-1,5,5-trimethyl-cyclohexene

$\sum($ Area. $\%)=$

Furans

Benzofuran

Benzofuran, 2-methyl

Furan, 2-(2 furanylmethyl)-5-methyl

Benzofuran, 4,7-dimethyl

$\sum($ Area. $\%)=$

Aldehyds

Myrtenal

Cinnamaldehyde

$\sum($ Area. $\%)=$

$\begin{array}{ccc}5.906 & 543-49-7 & 0.366 \\ 16.583 & 2490-48-4 & 0.849 \\ 10.263 & 99-48-9 & 1.259 \\ 9.760 & 4254-29-9 & 0.568 \\ 10.484 & - & 1.505 \\ 13.388 & 3344-45-4 & 0.427 \\ 12.300 & 132316-80-4 & 1.073 \\ & & 6.047 \\ & & \\ 8.816 & & 3.746 \\ 10.838 & 271-89-6 & 4.997 \\ 11.922 & 4265-25-2 & 1.209 \\ 12.739 & 13678-51-8 & 3.287 \\ & 28715-26-6 & \mathbf{1 3 . 2 3 9} \\ 10.034 & & \\ 12.654 & & 1.724 \\ & & 5.523 \\ & & \end{array}$

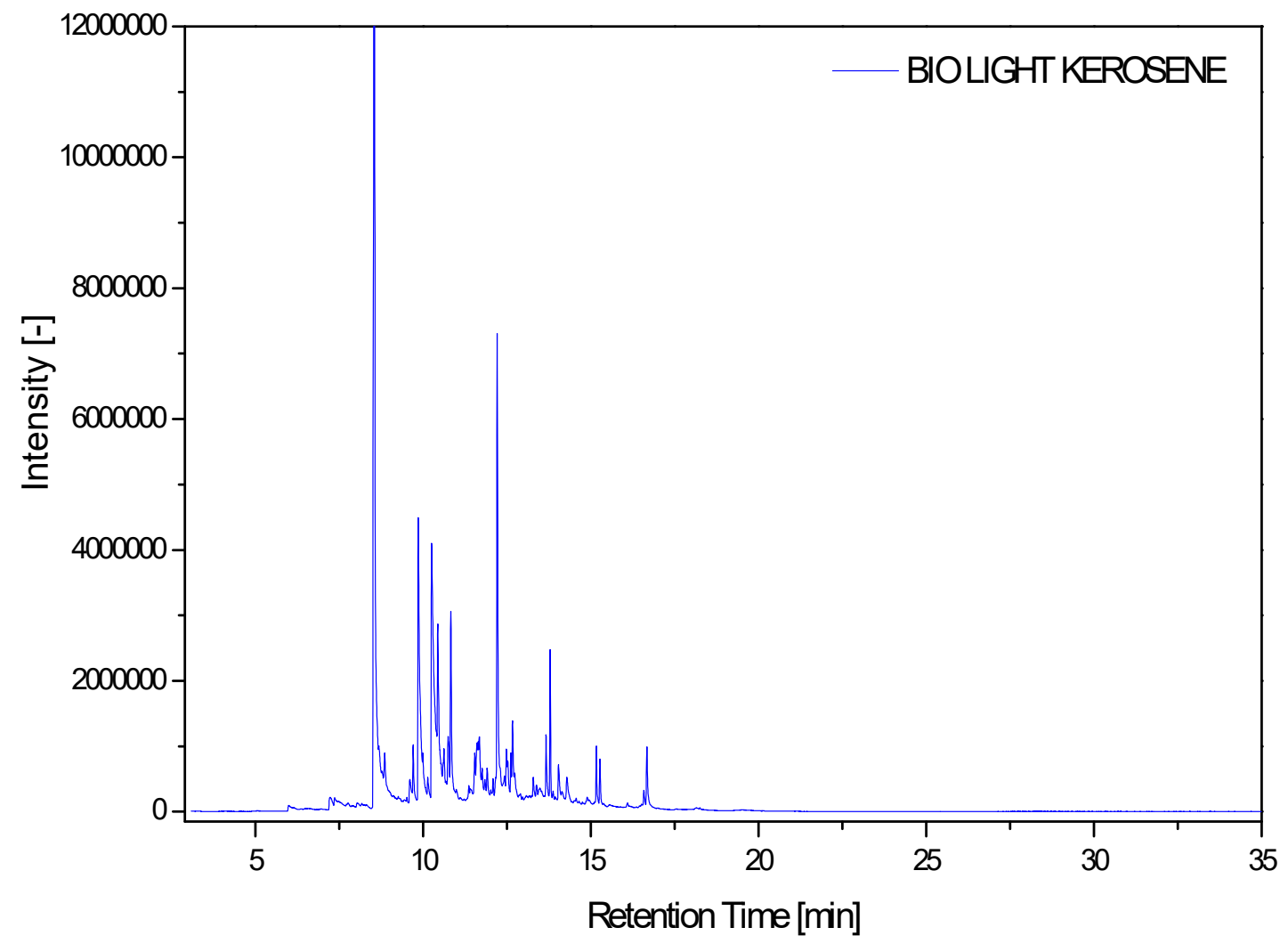

Figure 5: GC-MS of light kerosene-like fraction $\left(175^{\circ} \mathrm{C}-200^{\circ} \mathrm{C}\right)$.

The GC-MS identified in light kerosene-like fraction hydrocarbons (alkanes, alkenes, and aromatic hydrocarbons) and oxygenates (esters, carboxylic acids, phenols, alcohols, ketones, furans, and aldehydes). The light kerosene-like fraction is composed of $66.67 \%$ (area.) hydrocarbons (17.60\% alkenes, 32.65\% alkanes, and 16.42\% aromatic hydrocarbons) and 
$33.33 \%$ (area.) oxygenates $(6.16 \%$ esters, $4.24 \%$ ketones, $3.26 \%$ carboxylic acids, $7.13 \%$ phenols, $8.30 \%$ alcohols, $2.39 \%$ furans, and $1.86 \%$ aldehydes). The presence of carboxylic acids, ketones, furans, and phenols is associated to the high acidity of light kerosene-like fraction, as shown in Table 6.

Table 6: Classes of compounds, summation of peak areas, CAS number, and retention times of chemical compounds identified by CG-MS in light kerosene-like fraction $\left(175^{\circ} \mathrm{C}-200^{\circ} \mathrm{C}\right)$.

\begin{tabular}{|c|c|c|c|}
\hline Class of Compounds: Chemical Compounds & RT [min] & CAS & $\omega_{\mathrm{i}} \%$ (Area) \\
\hline \multicolumn{4}{|l|}{ Alkanes } \\
\hline Undecane & 10.558 & $1120-21-4$ & 2.115 \\
\hline Dodecane & 12.222 & $112-40-3$ & 1.608 \\
\hline Tridecane & 13.793 & $629-50-5$ & 7.177 \\
\hline Tetradecane & 15.266 & $629-59-4$ & 2.550 \\
\hline Pentadecane & 16.668 & $629-62-9$ & 8.424 \\
\hline Hexadecane & 17.987 & $544-76-3$ & 1.236 \\
\hline Heptadecane & 19.266 & $629-78-7$ & 8.974 \\
\hline Tetradacane 2,6,10-trimethyl- & 20.485 & $14905-56-7$ & 0,562 \\
\hline \multirow{2}{*}{\multicolumn{4}{|c|}{ Alkenes }} \\
\hline & & & \\
\hline 1-Dodecene & 12.096 & $112-41-4-6$ & 2.077 \\
\hline 1-Tridecene & 13.668 & 2437-56-1 & 1.225 \\
\hline 1-Tetradecene & 15.157 & $1120-36-1$ & 1.295 \\
\hline 1-Pentadecene & 16.576 & $13360-61-7$ & 3.279 \\
\hline 1-Heptadecene & 17.896 & $6765-39-5$ & 1.105 \\
\hline 3-Heptadecene,(Z)- & 18.991 & - & 2.914 \\
\hline 8-Heptadecene & 19.060 & 2579-04-6 & 5.701 \\
\hline$\sum($ Area. $\%)=$ & & & 17.596 \\
\hline \multicolumn{4}{|l|}{ Aromatic Hydrocarbons } \\
\hline Naphthalene, 2-methyl- & 14.023 & $91-57-6$ & 1.141 \\
\hline Naphthalene, 1-methyl- & 14.265 & $90-12-0$ & 1.582 \\
\hline Benzocycloheptatriene & 14.923 & $264-09-5$ & 1.633 \\
\hline Naphthalene, 1-ethyl- & 15.483 & $1127-76-0$ & 2.301 \\
\hline Naphthalene, 1,3-dimethyl- & 15.679 & $575-41-7$ & 3.739 \\
\hline Naphthalene, 1-(2-propenyl)- & 16.732 & $2489-86-3$ & 1.010 \\
\hline Naphthalene, 2-ethyl- & 16.806 & $827-54-3$ & 1.997 \\
\hline 1-Isopropenylnaphthalene & 17.145 & $1855-47-6$ & 0.848 \\
\hline Fluorene & 18.197 & $86-73-7$ & 1.722 \\
\hline 9H-Fluorene,9-methyl- & 18.415 & $2523-37-7$ & 0.445 \\
\hline$\sum($ Area. $\%)=$ & & & 16.418 \\
\hline \multicolumn{4}{|l|}{ Esters } \\
\hline Dodecanoic acid, methyl ester & 16.987 & $111-82-0$ & 3.801 \\
\hline Methyl tetradecanoate & 19.580 & $124-10-7$ & 2.358 \\
\hline$\Sigma($ Area.$\%)=$ & & & 6.159 \\
\hline \multicolumn{4}{|l|}{ Carboxylic Acids } \\
\hline 4,5-Dichlorothiophene-2-carboxylic acid & 14.766 & 31166-29-7 & 1.198 \\
\hline Erucic acid & 18.864 & $112-86-7$ & 0.450 \\
\hline Propanoic acid, 2-methyl-, (dodecahydro-6a-hydroxy- & 19.435 & $33649-17-1$ & 0.925 \\
\hline \multicolumn{4}{|l|}{ 9a-methylene-2,9-dioxoazuleno } \\
\hline Cis-5,8,11,14,17-Eicosapentaenoic acid & 20.403 & $10417-94-4$ & 0.692 \\
\hline$\sum($ Area.$\%)=$ & & & 3.265 \\
\hline \multicolumn{4}{|l|}{ Ketones } \\
\hline $\begin{array}{l}\text { Cyclopenta[1,3]cyclopropa[1,2]cyclohepten-3(3aH)- } \\
\text { one, } 1,2,3 \mathrm{~b}, 6,7,8 \text {-hexahydro }\end{array}$ & 14.851 & $91531-58-7$ & 1.013 \\
\hline $\begin{array}{l}\text { 4-(2,4,4-Trimethyl-cyclohexa-1,5-dienyl)-but-3-en-2- } \\
\text { one }\end{array}$ & 15.980 & - & 0.966 \\
\hline $\begin{array}{l}\text { Cyclopenta[1,3]cyclopropa[1,2]cyclohepten-3(3aH)- } \\
\text { one, } 1,2,3 \mathrm{~b}, 6,7,8 \text {-hexahydro-1,2,3b }\end{array}$ & 16.330 & $91531-58-7$ & 0.879 \\
\hline $\begin{array}{l}\text { 2,4,6-Cycloheptatrien-1-one,2-hydroxy-5-(3-methyl-2- } \\
\text { butenyl)-4-(1-methylethenyl)- }\end{array}$ & 16.887 & $552-96-5$ & 1.382 \\
\hline
\end{tabular}


$\sum($ Area. $\%)=$

Phenols

Phenol, 2,5-dimethyl-

Phenol, 3,4-dimethyl-

Phenol, 3-ethyl-5-methyl-

$\sum($ Area.$\%)=$

Alcohols

9-Methyltricyclo[4.2.1.1(2,5)]deca-3,7-diene-9,10-diol

1-Naphthalenol, 1,2,3,4-tetrahydro-2,5,8-trimethyl-

Bicyclo[4.1.0]heptan-2-ol,1 $\beta$-(3-methyl-1,3-

butadienyl)-2 $\alpha, 6 \beta$-dimethyl-3 $\beta$-acetoxy

Cyclopentanol, 3,3,4-trimethyl-4-p-tolyl-, (R,R)-(+)-

1-Hexadecanol, 2-methyl-

$\sum($ Area. $\%)=$

\section{Furans}

Dibenzofuran

$\sum($ Area. $\%)=$

Aldehyds

2-((2R,4aR,8aS)-4a-Methyl-8-

methylenedecahydronaphthalen-2-yl)acrylaldehyde

$\Sigma($ Area.$\%)=$

$\begin{array}{lcc} & & \mathbf{4 . 2 4 0} \\ 11.725 & 95-87-4 & 1.168 \\ 12.015 & 95-65-8 & 1.274 \\ 13.591 & 698-71-5 & 4.686 \\ & & \mathbf{7 . 1 2 8} \\ 14.176 & 78323-73-6 & 0.789 \\ 16.162 & 55591-08-7 & 1.862 \\ 16.378 & - & 2.696 \\ & & \\ 16.475 & 19902-38-6 & 1.604 \\ 19.172 & 2490-48-4 & 1.348 \\ & & \mathbf{8 . 2 9 9} \\ 17.299 & 132-64-9 & 2.387 \\ & & \mathbf{2 . 3 8 7} \\ 17.791 & 3650-40-6 & 1.864 \\ & & \mathbf{1 . 8 6 4}\end{array}$

By the GC-MS analysis of kerosene-like fraction, hydrocarbons (alkanes, alkenes, and aromatic hydrocarbons) and oxygenates (esters, ethers, phenols, alcohols, ketones, furans, and aldehydes) were identified.

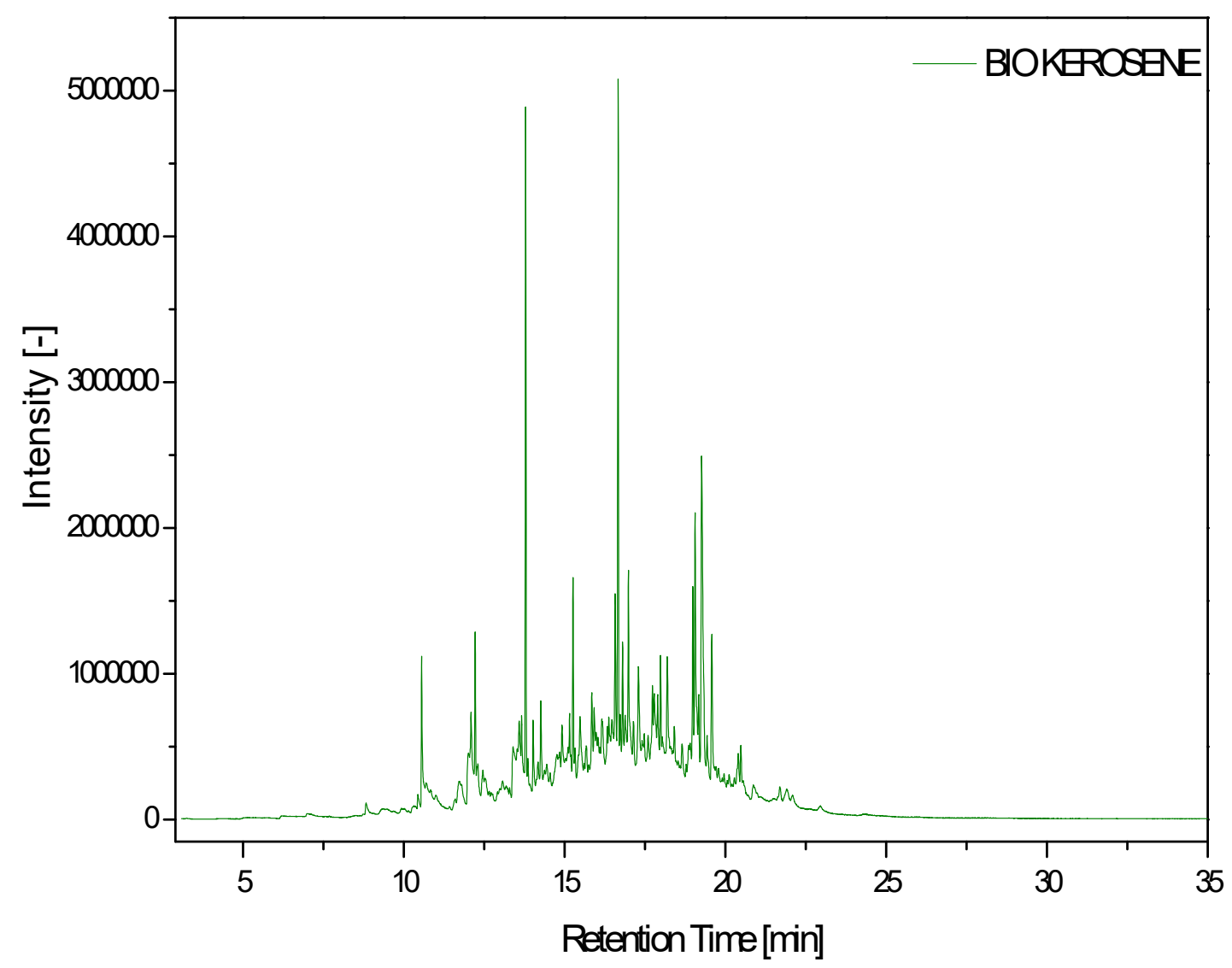

Figure 6: GC-MS of kerosene-like fraction $\left(200^{\circ} \mathrm{C}-215^{\circ} \mathrm{C}\right)$. 
The kerosene-like fraction is composed of $19.87 \%$ (area.) hydrocarbons $(2.79 \%$ alkenes, $4.20 \%$ alkanes, and $12.88 \%$ aromatic hydrocarbons) and $81.13 \%$ (area.) oxygenates $(2.06 \%$ esters, $0.80 \%$ ethers, $3.50 \%$ ketones, $60.79 \%$ phenols, $0.96 \%$ alcohols, $8.99 \%$ furans, and $3.22 \%$ aldehydes). The presence of ketones, furans, ethers, esters, aldehydes, and phenols confer the high acidity of kerosene-like fraction, as summarized in Table 7. Finally, the content of hydrocarbons within the distillation fractions (gasoline: $40^{\circ} \mathrm{C}<\mathrm{T}^{\text {Boiling }}<175^{\circ} \mathrm{C}$; light kerosene: $175^{\circ} \mathrm{C}<\mathrm{T}^{\text {Boiling }}<200^{\circ} \mathrm{C}$; and kerosene-like fraction: $200^{\circ} \mathrm{C}<\mathrm{T}^{\text {Boiling }}<215^{\circ} \mathrm{C}$ ) are higher than those reported in the literature [17-19, 46-48, 66, 70, 72], showing that was effective not only to diminish the acidity, but also to concentrate hydrocarbons.

Table 7: Classes of compounds, summation of peak areas, CAS number, and retention times of chemical compounds identified by CG-MS in kerosene-like fraction $\left(200^{\circ} \mathrm{C}-215^{\circ} \mathrm{C}\right)$.

\begin{tabular}{|c|c|c|c|}
\hline Class of Compounds: Chemical Compounds & RT [min] & CAS & $\omega_{i} \%$ (Area) \\
\hline \multicolumn{4}{|l|}{ Alkanes } \\
\hline Tridecane & 13.792 & $629-50-5$ & 2.023 \\
\hline Tetradecane & 15.276 & $629-59-4$ & 0.752 \\
\hline Pentadecane & 16.675 & $629-62-9$ & 1.422 \\
\hline$\sum($ Area. $\%)=$ & & & 4.20 \\
\hline \multicolumn{4}{|l|}{ Alkenes } \\
\hline Tetracyclo[5.3.0.0<2,6>.0<3,10>]deca-4,8-diene & 11.546 & $34324-40-8$ & $0,981 \%$ \\
\hline Bicyclo[6.4.0]dodeca-9,11-diene & 13.288 & - & $0,389 \%$ \\
\hline 1-Tetradecene & 15.166 & $1120-36-1$ & $1,027 \%$ \\
\hline 1-Pentadecene & 16.586 & $13360-61-7$ & $0,390 \%$ \\
\hline$\sum($ Area.\% $)=$ & & & 2.787 \\
\hline \multicolumn{4}{|l|}{ Aromatic Hydrocarbons } \\
\hline Benzene, 1-ethynyl-4-methyl- & 9.708 & $766-97-2$ & 1.148 \\
\hline 1H-Indene, 2,3-dihydro-4-methyl- & 11.370 & $824-22-6$ & 0.288 \\
\hline Naphthalene & 12.213 & $91-20-3$ & 9.719 \\
\hline Naphthalene, 1-methyl & 14.043 & $90-12-0$ & 0.842 \\
\hline Naphthalene, 2-methyl- & 14.290 & $91-57-6$ & 0.883 \\
\hline$\sum($ Area.\% $)=$ & & & 12.880 \\
\hline \multicolumn{4}{|l|}{ Alcohol } \\
\hline 1,3-Cyclohexadiene-1-methanol, $\alpha, 2,6,6$-tetramethyl- & 11.764 & 102676-97-1 & 0.773 \\
\hline 9-Heptadecene-4,6-diyn-3-ol & 13.386 & $1242413-82-6$ & 0.187 \\
\hline$\sum($ Area. $\%)=$ & & & 0.960 \\
\hline \multicolumn{4}{|l|}{ Ether } \\
\hline p-Propargyloxytoluene & 12.431 & $5651-90-1$ & 0.803 \\
\hline$\sum($ Area.\% $\%=$ & & & 0.803 \\
\hline \multicolumn{4}{|l|}{ Ketones } \\
\hline 2-Cyclopenten-1-one, 2-methyl- & 7.237 & $1120-73-6$ & 0.447 \\
\hline Ethanone, 1-(2-furanyl)- & 7.366 & $1192-62-7$ & 0.178 \\
\hline 2-Cyclopenten-1-one, 2,3-dimethyl & 9.609 & $1121-05-7$ & 0.735 \\
\hline Benzoin & 10.144 & $119-53-9$ & 0.511 \\
\hline 8-Decen-2-one, 9-methyl-5-methylene- & 12.090 & $130876-97-0$ & 0.354 \\
\hline $\begin{array}{l}\text { Bicyclo[8.2.0]dodecan-11-one, 12,12-dichloro-, } \\
\left(1 \mathrm{R}^{*}, 10 \mathrm{~S}^{*}\right) \text { - }\end{array}$ & 13.672 & 110079-11-3 & 1.078 \\
\hline$\sum($ Area. $\%)=$ & & & 3.503 \\
\hline \multicolumn{4}{|l|}{ Phenols } \\
\hline Phenol & 8.860 & $108-95-2$ & 31.258 \\
\hline Phenol, 2-methyl- & 9.861 & $95-48-7$ & 8.621 \\
\hline Phenol, 3-methyl- & 9.995 & $108-39-4$ & 13.132 \\
\hline Phenol, 2-methoxy & 10.442 & $90-05-1$ & 5.554 \\
\hline Phenol, 2,5-dimethyl & 11.645 & $95-87-4$ & 2.229 \\
\hline$\sum($ Area. $\%)=$ & & & 60.794 \\
\hline
\end{tabular}




\begin{tabular}{|c|c|c|c|}
\hline \multicolumn{4}{|l|}{ Ester } \\
\hline $\begin{array}{l}\text { 1-hydroxy-1,2,3,4-tetrahydronaphthalene } \\
\text { trifluoroacetate ester }\end{array}$ & 11.846 & $134563-46-5$ & 2.065 \\
\hline$\sum($ Area.\% $)=$ & & & 2.065 \\
\hline \multicolumn{4}{|l|}{ Furans } \\
\hline Benzofuran, 2-methyl- & 10.831 & $4265-25-2$ & 3.546 \\
\hline Furan, 2-(2 furanylmethyl)-5-methyl- & 11.914 & $13678-51-8$ & 0.750 \\
\hline Benzofuran, 4,7-dimethyl- & 12.486 & $28715-26-6$ & 4.693 \\
\hline $\begin{array}{l}\sum(\text { Area.\% })= \\
\text { Aldehyds }\end{array}$ & & & 8.989 \\
\hline 2-Propenal, 3-phenyl- & 10.753 & $104-55-2$ & 3.219 \\
\hline$\sum($ Area.\% $)=$ & & & 3.219 \\
\hline
\end{tabular}

\section{Conclusions}

The yield of distillation fractions (gasoline, light kerosene, and kerosene-like like fractions), $77.61 \%$ (wt.), is higher but according than those reported in the literature for both atmospheric and vacuum conditions [17-19, 21, 41, 46-48, 53, 66, 70, 72]. The acid values of distillation fractions increase with increasing boiling temperature. However, the acidity of gasoline-like fraction is much lower than that of raw bio-oil $(70.26 \mathrm{mg} \mathrm{KOH} / \mathrm{g})$. The same behavior was observed for the densities, kinematic viscosities, and refractive indexes of gasoline, light kerosene, and kerosene-like like fractions with increasing boiling temperature.

The FT-IR analysis of bio-oil and distillation fraction identify the presence of hydrocarbons (alkanes, alkenes, and aromatic hydrocarbons) and oxygenates (phenols, cresols, carboxylic acids, alcohols, ethers, ketones, and furans). The bio-oil is composed of $21.52 \%$ (area) hydrocarbons and $78.48 \%$ (area) oxygenates. The presence of carboxylic acids, as well as phenols and cresols is associated to the high acidity of bio-oil.

The gasoline-like fraction is composed by $64.0 \%$ (area.) hydrocarbons and $36.0 \%$ (area.) oxygenates, while light kerosene-like fraction by $66.67 \%$ (area.) hydrocarbons and $33.33 \%$ (area.) oxygenates, and kerosene-like fraction by 19.87\% (area.) hydrocarbons and $81.13 \%$ (area.) oxygenates. The content of hydrocarbons within the distillation fractions are higher than those reported in the literature [17-19, 46-48, 66, 70, 72], showing that distillation was effective not only to diminish the acidity, but also to concentrate hydrocarbons.

\section{References}

[1] Jonny Everson Scherwinski-Pereira; Rodrigo da Silva Guedes; Ricardo Alexandre da Silva; Paulo César Poeta Fermino Jr.; Zanderluce Gomes Luis; Elínea de Oliveira Freitas. Somatic embryogenesis and plant regeneration in açaí palm (Euterpe oleracea). Plant Cell Tiss Organ Cult (2012) 109:501-508, DOI 10.1007/s11240-012-0115-z 
[2] Alexander G. Schauss; Xianli Wu; Ronald L. Prior; Boxin Ou; Dinesh Patel; Dejian Huang; James P. Kababick. Phytochemical and Nutrient Composition of the Freeze-Dried Amazonian Palm Berry, Euterpe oleraceae Mart. (Acai). J. Agric. Food Chem. 2006, 54, 22, 8598-8603

[3] Sara Sabbe; Wim Verbeke; Rosires Deliza; Virginia Matta; Patrick Van Damme. Effect of a health claim and personal characteristics on consumer acceptance of fruit juices with different concentrations of açaí (Euterpe oleracea Mart.). Appetite 53 (2009) 84-92, doi: 10.1016/j.appet.2009.05.014

[4] Lisbeth A. Pacheco-Palencia; Christopher E. Duncan; Stephen T. Talcott. Phytochemical composition and thermal stability of two commercial açai species, Euterpe oleracea and Euterpe precatoria. Food Chem. 115 (2009) 1199-1205, doi: 10.1016/j.foodchem.2009.01.034

[5] Eduardo S. Brondízio; Carolina A. M. Safar; Andréa D. Siqueira. The urban market of Açaí fruit (Euterpe oleracea Mart.) and rural land use change: Ethnographic insights into the role of price and land tenure constraining agricultural choices in the Amazon estuary. Urban Ecosystems (2002) 6-67, https://doi.org/10.1023/A:1025966613562

[6] Elisabeth dos Santos Bentes; Alfredo Kingo Oyama Homma; César Augusto Nunes dos Santos. Exportações de Polpa de Açaí do Estado do Pará: Situação Atual e Perspectivas. In: Anais Congresso da Sociedade Brasileira de Economia, Administração e Sociologia Rural, 55, Santa Maria, RS-Brazil, 2017, https://www.researchgate.net/publication/319465735 Exportacoes de Polpa de Acai do Es tado_do_Para_Situacao_Atual_e_Perspectivas.

[7] Ana Victoria da Costa Almeida; Ingrid Moreira Melo; Isis Silva Pinheiro; Jessyca Farias Freitas; André Cristiano Silva Melo. Revalorização do caroço de açaí em uma beneficiadora de polpas do município de Ananindeua/PA: proposta de estruturação de um canal reverso orientado pela PNRS e logística reversa. GEPROS. Gestão da Produção, Operações e Sistemas, Bauru, Ano 12, No 3, jul-set/2017, 59-83. DOI: 10.15675/gepros.v12i3.1668

[8] Claudio Ramalho Townsend; Newton de Lucena Costa; Ricardo Gomes de Araújo Pereira; Clóvis C. Diesel Senger. Características químico-bromatológica do caroço de açaí. COMUNICADO TÉCNICO No 193 (CT/193), EMBRAPA-CPAF Rondônia, ago./01, 1-5. ISSN 0103-9458, https://ainfo.cnptia.embrapa.br/digital/bitstream/item/100242/1/Cot193acai.pdf

[9] Carlos Fioravanti. Açaí: Do pé para o lanche. Revista Pesquisa Fapesp, Vol. 203, Janeiro de 2013, 64-68, http://revistapesquisa.fapesp.br/2013/01/11/folheie-a-edicao-203/ 
[10] Antônio Cordeiro de Santana; Ádamo Lima de Santana; Ádina Lima de Santana; Marcos Antônio Souza dos Santos; Cyntia Meireles de Oliveira. Análise Discriminante Múltipla do Mercado Varejista de Açaí em Belém do Pará. Rev. Bras. Frutic., Jaboticabal - SP, Vol. 36, N. 3, 532- 541, Setembro 2014, http://dx.doi.org/10.1590/0100-2945-362/13

[11] José Dalton Cruz Pessoa; Paula Vanessa da Silva e Silva. Effect of temperature and storage on açaí (Euterpe oleracea) fruit water uptake: simulation of fruit transportation and preprocessing. Fruits, 2007, Vol. 62, 295-302; DOI: 10.1051/fruits:2007025 www.fruitsjournal.org

[12] Cordeiro M. A. Estudo da hidrólise enzimática do caroço de açaí (Euterpe oleracea, Mart) para a produção de etanol. Dissertação de Mestrado, Programa de Pós-Graduação em Engenharia Química, UFPA-Brazil. Marcio de Andrade Cordeiro; 2016

[13] Tamiris Rio Branco da Fonseca; Taciana de Amorim Silva; Mircella Marialva Alecrim; Raimundo Felipe da Cruz Filho; Maria Francisca Simas Teixeira. Cultivation and nutritional studies of an edible mushroom from North Brazil. African Journal of Microbiology Research. 2015;9(30):1814-1822

[14] Kababacknik A; Roger H. Determinação do poder calorífico do caroço do açaí em três distintas umidades, $38^{\text {th }}$ Congresso Brasileiro de Química, São Luiz-MA-Brazil; 1998

[15] Altman R. F. A. O Caroço de açaí (Euterpe oleracea, Mart). Vol. 31. Belém-Pa, Brasil: Boletim Técnico do Instituto Agronômico do Norte; 1956, 109-111

[16] Diadem Özçimen; Ayşegül Ersoy-Meriçboyu. Characterization of biochar and bio-oil samples obtained from carbonization of various biomass materials. Renewable Energy, June 2010;35(6):1319-1324

[17] John D. Adjaye; Ramesh K. Sharma; Narendra N. Bakhshi. Characterization and stability analysis of wood-derived bio-oil. Fuel Processing Technology 31 (1992) 241-256

[18] Carazza F; Rezende M. E. A; Pasa V. M. D; Lessa A. Fractionation of wood tar. Proc Adv Thermochem Biomass Convers 1994; 2:465

[19] J. D. Adjaye; N. N. Bakhshi. Production of hydrocarbons by catalytic upgrading of a fast pyrolysis bio-oil. Part I: Conversion over various catalysts. Fuel Processing Technology 45 (1995) 161-183 
[20] Xu B. J; Lu N. Experimental research on the bio-oil derived from biomass pyrolysis liquefaction. Trans Chin Soc Agr Eng 1999;15:177-81

[21] Boucher M. E; Chaala A; Roy C. Bio-oils obtained by vacuum pyrolysis of softwood bark as a liquid fuel for gas turbines. Part I: Properties of bio-oil and its blends with methanol and a pyrolytic aqueous phase. Biomass Bioenergy 2000;19:337-50

[22] Oasmaa A; Kuoppala E; Gust S; Solantausta Y. Fast pyrolysis of forestry residue. 1. Effect of extractives on phase separation of pyrolysis liquids. Energy \& Fuels. 2003;17(1): 1-12

[23] Czernik S; Bridgwater A. V. Overview of applications of biomass fast pyrolysis oil. Energy \& Fuels. 2004;18:590-598

[24] Mohan D; Pittman C. U. Jr; Steelee P. H. Pyrolysis of wood/biomass for bio-oil: A critical review. Energy \& Fuels. 2006; 20:848-889

[25] Fei Yu; Shaobo Deng; Paul Chen; Yuhuan Liu; Yiquin Wan; Andrew Olson; David Kittelson; Roger Rua. Physical and Chemical Properties of Bio-Oils From Microwave Pyrolysis of Corn Stover. Applied Biochemistry and Biotechnology 136-140 (2007) 957-970

[26] Zhang Qi; Chang Jie; Wang Tiejun; Xu Ying. Review of biomass pyrolysis oil properties and upgrading research. Energy Conversion and Management 48 (2007) 87-92

[27] Boateng A. A; Mullen C. A; Goldberg N; Hicks K. B. Production of bio-oil from alfalfa stems by fluidized-bed fast pyrolysis. Industrial and Engineering Chemistry Research. $2008 ; 47: 4115-4122$

[28] Lu Qiang; Yang Xu-lai; ZhuXi-feng. Analysis on chemical and physical properties of biooil pyrolyzed from rice husk. Journal of Analytical and Applied Pyrolysis 82 (2008) 191-198

[29] Xu Junming; Jiang Jianchun; SunYunjuan; LuYanju. Bio-Oil Upgrading by means of Ethyl Ester Production in Reactive Distillation to Remove Water to Improve Storage and Fuel Characteristics. Biomass and Bioenerg 32 (2008) 1056-1061

[30] Guo Z.; Wang S.; Zhu Y.; Luo Z.; Cen K. Separation of acid compounds for refining biomass pyrolysis oil. Journal of Fuel Chemistry and Technology. 2009;7(1):49-52

[31] Vispute T. P; Huber G. W. Production of hydrogen, alkanes and polyols by aqueous phase processing of wood-derived pyrolysis oils. Green Chemistry. 2009;11:1433-1445

[32] Song Q; Nie J; Ren M; Guo Q. Effective phase separation of biomass pyrolysis oils by adding aqueous salt solutions. Energy \& Fuels. 2009;23:3307-3312 
[33] Shurong Wang;; Yueling Gu; Qian Liu; YanYao; Zuogang Guo; Zhongyang Luo; Kefa Cen. Separation of bio-oil by molecular distillation. Fuel Processing Technology 90 (2009) 738-745 [34] Oasmaa A; Elliott D. C; Korhonen J. Acidity of biomass fast pyrolysis bio-oils. Energy \& Fuels. 2010;24(12):6548-6554

[35] Michael W. Nolte; Matthew W. Liberatore. Viscosity of Biomass Pyrolysis Oils from Various Feedstocks. Energy Fuels 2010, 24, 12, 6601-6608

[36] Guo X.; Wang S.; Guo Z.; Liu Q.; Luo Z.; Cen K. Pyrolysis characteristics of bio-oil fractions separated by molecular distillation. Applied Energy. 2010;87(9):2892-2898

[37] Guo Z.; Wang S.; Gu Y.; Xu G.; Li X.; Luo Z. Separation characteristics of biomass pyrolysis oil in molecular distillation. Separation and purification. 2010;76(1):52-57

[38] Xiujuan Guo; Shurong Wang; Zuogang Guo; Qian Liu; Zhongyang Luo; Kefa Cen. Pyrolysis characteristics of bio-oil fractions separated by molecular distillation. Applied Energy 87 (2010) 2892-2898

[39] Zuogang Guo; Shurong Wang; Yueling Gu; Guohui Xu; Xin Li; Zhongyang Luo. Separation characteristics of biomass pyrolysis oil in molecular distillation. Separation and Purification Technology 76 (2010) 52-57

[40] Christensen E. D; Chupka G. M; Smurthwaite J. L. T; Alleman T. L; Lisa K; Franz J. A; Elliott D. C; Mc Cormick R. L. Analysis of oxygenated compounds in hydrotreated biomass fast pyrolysis oil distillate fractions. Energy \& Fuels. 2011;25(11):5462-5471

[41] Ji-Lu Zheng; Qin Wei. Improving the quality of fast pyrolysis bio-oil by reduced pressure distillation. Biomass and Bioenergy 35 (2011) 1804-1810

[42] A. S. Pollard; M. R. Rover; R. C. Brown. Characterization of bio-oil recovered as stage fractions with unique chemical and physical properties. Journal of Analytical and Applied Pyrolysis 93 (2012) 129-138

[43] Ajay Shah; Matthew J. Darr; Dustin Dalluge; Dorde Medic; Keith Webster; Robert C. Brown. Physicochemical properties of bio-oil and biochar by fast pyrolysis of stored singlepass corn Stover and cobs. Bioresource Technology 125 (2012) 348-352

[44] Tahmina Imam; Sergio Capareda. Characterization of bio-oil, syn-gas and bio-char from switch grass pyrolysis at various temperatures. Journal of Analytical and Applied Pyrolysis 93 (2012) 170-177 
[45] Shuangning Xiu; Abolghasem Shahbazi. Bio-oil production and upgrading research. A Review. Renewable and Sustainable Energy Reviews 16 (2012) 4406-4414

[46] Arakshita Majhi; Y. K. Sharma; D. V. Naik. Blending optimization of Hempel distilled bio-oil with commercial diesel. Fuel 96 (2012) 264-269

[47] Xue-Song Zhang; Guang-Xi Yang; Hong Jiang; Wu-Jun Liu; Hong-Sheng Ding. Mass production of chemicals from biomass-derived oil by directly atmospheric distillation coupled with co-pyrolysis. Scientific Reports. 2013;3:1-7. Article Number 1120

[548] Jewel A. Capunitan; Sergio C. Capareda. Characterization and separation of corn stover bio-oil fractional distillation. Fuel 112 (2013) 60-73

[49] Yining Sun; Bin Gao; YingYao; June Fang; Ming Zhang; Yanmei Zhou; Hao Chen; Liuyan Yang. Effects of feedstock type, production method, and pyrolysis temperature on biochar and hydrochar properties. Chemical Engineering Journal 240 (2014) 574-578

[50] Huijun Yang; Jingang Yao; Guanyi Chen; Wenchao Ma; BeibeiYan; YunQi. Overview of upgrading of pyrolysis oil of biomass. Energy Procedia 61 (2014) 1306-1309

[51] Akhil Tumbalam Gooty; Dongbing Li; Franco Berruti; Cedric Briens. Kraft-lignin pyrolysis and fractional condensation of its bio-oil vapors. Journal of Analytical and Applied Pyrolysis 106 (2014) 33-40

[52] C. H. Biradar; K. A. Subramanian; M. G. Dastidar. Production and fuel upgrading of pyrolysis bio-oil Jatropha Curcas de-oiled seed cake. Fuel 119 (2014) 81-89

[53] Elkasabi Y; Mullen C. A; Boateng A. A. Distillation and isolation of commodity chemicals from bio-oil made by tail-gas reactive pyrolysis. Sustainable. Chem. Eng. 2014;2:2042-2052

[54] Akhil Tumbalam Gooty; Dongbing Li; Cedric Briens; Franco Berruti. Fractional condensation of bio-oil vapors produced from birch bark pyrolysis. Separation and Purification Technology 124 (2014) 81-88

[55] Shurong Wang; Qinjie Cai; Xiangyu Wang; Li Zhang; Yurong Wang; Zhongyang Luo. Biogasoline production from the co-cracking of the distilled fraction of bio-oil and ethanol. Energy Fuels, 2014, 28 (1), 115-122

[56] Sadegh Papari; Kelly Hawboldt. A review on the pyrolysis of woody biomass to bio-oil: Focus on kinetic models. Renewable and Sustainable Energy Reviews 52 (2015) 1580-1595 
[57] Harpreet Singh; Kambo Animesh Dutta. A comparative review of biochar and hydro-char in terms of production, physico-chemical properties and applications. Renewable and Sustainable Energy Reviews 45(2015) 359-378

[58] Shushil Kumar; Jean-Paul Lange; Guus Van Rossum; Sascha R. A. Kersten. Bio-oil fractionation by temperature-swing extraction: Principle and application. Biomass and Bioenergy 83 (2015) 96-104

[59] Yaseen Elkasabi; Akwasi A. Boateng; Michael A. Jackson. Upgrading of bio-oil distillation bottoms into biorenewable calcined coke. Biomass and Bioenergy 81 (2015) 415 423

[60] Yaseen Elkasabi; Charles A. Mullen; Michael A. Jackson; Akwasi A. Boateng. Characterization of fast-pyrolysis bio-oil distillation residues and their potential applications. Journal of Analytical and Applied Pyrolysis 114 (2015) 179-186

[61] Kanaujia P. K; Naik D. V; Tripathi D; Singh R; Poddar M. K; Siva Kumar Konathala L. N; Sharma Y. K. Pyrolysis of Jatropha Curcas seed cake followed by optimization of liquidliquid extraction procedure for the obtained bio-oil. Anal. Appl. Pyrolysis. 2016;118:202-224 [62] Tao Kan; Vladimir Strezov; Tim J. Evans. Lignocellulose biomass pyrolysis: A review of product properties and effects of pyrolysis parameters. Renewable and Sustainable Energy Reviews 57 (2016) 1126-1140

[63] Junmeng Cai; Scott W. Banks; Yang, Surila Darbar; Tony Bridgwater. Viscosity of Aged Bio-Oils from Fast Pyrolysis of Beech Wood and Miscanthus: Shear Rate and Temperature Dependence. Energy Fuels 2016, 30, 6, 4999-5004

[64] Yunwu Zheng; Fei Wang; Xiaoqin Yang; Yuanbo Huang; Can Liu; Zhifeng Zheng; Jiyou $\mathrm{Gu}$. Study on aromatics production via catalytic pyrolysis vapor upgrading of biomass using metal-loaded modified H-ZSM-5. Journal of Analytical and Applied Pyrolysis 126 (2017) 169179

[65] Ann Christine Johansson; Kristiina Iisa; Linda Sandström; Haoxi Ben; Heidi Pilath; Steve Deutch; Henrik Wiinikka; Olov G. W. Öhrman. Fractional condensation of pyrolysis vapors produced from Nordic feedstocks in cyclone pyrolysis. Journal of Analytical and Applied Pyrolysis 123 (2017) 244-254 
[66] Hsiu-Po Kuo; Bo-Ren Hou; An-Ni Huang. The influence of the gas fluidization velocity on the properties of bio-oils from fluidized bed pyrolizer with in-line distillation. Applied Energy 194 (2017) 279-286

[67] Raquel Escrivani Guedes; Aderval S. Luna; Alexandre Rodrigues Torres. Operating parameters for bio-oil production in biomass pyrolysis: A review. Journal of Analytical and Applied Pyrolysis 129 (2018) 134-149

[68] Vaibhav Dhyani; Thallada Bhaskar. A comprehensive review on the pyrolysis of lignocellulosic biomass. Renewable Energy 129 (2018) 695-716

[69] Wenfei Cai; Ronghou Liu; Yifeng He; Meiyun Chai; Junmeng Cai. Bio-oil production from fast pyrolysis of rice husk in a commercial-scale plant with a downdraft circulating fluidized bed reactor. Fuel Processing Technology 171 (2018) 308-317

[70] Ni Huang; Chen-Pei Hsu; Bo-Ren Hou; Hsiu-Po Kuo. Production and separation of rice husk pyrolysis bio-oils from a fractional distillation column connected fluidized bed reactor. Powder Technology 323 (2018) 588-593

[71] Shofiur Rahman; Robert Helleur; Stephanie MacQuarrie; Sadegh Papari; Kelly Hawboldt. Upgrading and isolation of low molecular weight compounds from bark and softwood bio-oils through vacuum distillation. Separation and Purification Technology 194 (2018) 123-129

[72] An-Ni Huang; Chen-Pei Hsua; Bo-Ren Houa; Hsiu-Po Kuo. Production and separation of rice husk pyrolysis bio-oils from a fractional distillation column connected fluidized bed reactor. Powder Technology Volume 323, 1 January 2018, 588-593

[73] D. A. R. de Castroa; H. J. da Silva Ribeiro; C. C. Ferreira; L. H. H. Guerreiroa; M. de Andrade Cordeiro; A. M. Pereira; W. G. dos Santos; F. B. de Carvalho; J. O. C. Silva Jr.; R. Lopes e Oliveira; M. C. Santos; S. Duvoisin Jr; L. E. P. Borges; N. T. Machado. Fractional Distillation of Bio-Oil Produced by Pyrolysis of Açaí (Euterpe oleracea) Seeds. Editor Hassan Al-Haj Ibrahim: Fractionation, Intechopen ISBN: 978-1-78984-965-3, DOI: 10.5772/intechopen. 79546

[74] E. C. Costa; C. C. Ferreira; A. L. B. dos Santos; H. da Silva Vargens; E. G. O. Menezes; V. M. B. Cunha; M. P. da Silva; A. A. Mâncio; N. T. Machado; M. E. Araújo. Process simulation of organic liquid products fractionation in countercurrent multistage columns using $\mathrm{CO}_{2}$ as solvent with Aspen-Hysys. The Journal of Supercritical Fluids Volume 140, October 2018, 101-115 
[75] Standards T. Acid-Insoluble Lignin in Wood and Pulp. Tappi Method T 222 Om-06. Atlanta, GA: Tappi Press. 2006

[76] Buffiere P; Loisel D. Dosage des fibres Van Soest. Weened, Laboratoire de Biotechnologie de l'Environnement. INRA Narbonne. 2007:1-14

[77] da Mota S. A. P; Mâncio A. A; Lhamas D. E. L; de Abreu D. H; da Silva M. S; dos Santos W. G; de Castro D. A. R; de Oliveira R. M; Araújo M. E; Borges L. E. P; Machado N. T. Production of green diesel by thermal catalytic cracking of crude palm oil (Elaeis guineensis Jacq) in a pilot plant. Journal of Analytical and Applied Pyrolysis. 2014;110:1-11

[78] Ferreira C. C; Costa E. C; de Castro D. A. R; Pereira M. S; Mâncio A. A; Santos M. C; Lhamas D. E. L; da Mota S. A. P; Leão A. C; Duvoisin S. Jr; Araújo M. E; Borges L. E. P; Machado N. T. Deacidification of organic liquid products by fractional distillation in laboratory and pilot scales. Journal of Analytical and Applied Pyrolysis. 2017;127:468-489

[79] Tuya Ba. Abdelkader Chaala; Manuel Garcia-Perez; Denis Rodrigue; Christian Roy. Colloidal properties of bio-oils obtained by vacuum pyrolysis of softwood bark. Characterization of water-soluble and water-insoluble fractions. Energy Fuels 2004;18:704-12 [80] Sathish K. Tanneru; Divya R. Parapati; Philip H. Steele. Pretreatment of bio-oil followed by upgrading via esterification to boiler fuel. Energy 73 (2014) 214-220

[81] Seshadri K. S; Cronauer D. C. Characterization of coal-derived liquids by ${ }^{13}$ C N.M.R. and FT-IR Spectroscopy. Fuel. 1983;62:1436-1444

Thermodynamic Studies. J. Chem. Eng. Data 2016, 61, 213-219, DOI: 10.1021/acs.jced.5b00481 